\title{
Tranexamic acid after cardiopulmonary bypass and perioperative outcomes after cardiac surgery
}

Jing Liu, Changwei Chen, Lei Du

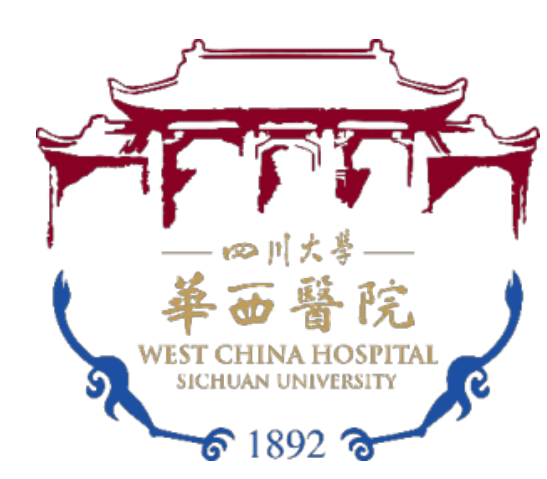

Department of Anaesthesiology, West China Hospital, Sichuan University, Chengdu, China

\section{BACKGROUND}

Tranexamic acid (TXA) has been widely used after induction of anaesthesia to reduce bleeding in cardiac surgery with cardiopulmonary bypass (CPB). Whereas, TXA was reported to be associated with increased incidence of thrombotic complications. To reduce the risk of adverse outcomes associated with TXA, we began administrating TXA after CPB since July 1, 2011. This retrospective study was to observe whether the administration of TXA after CPB could reduce postoperative complications.

\section{METHODS}

After the approval of ethics committee of West China Hospital (No. 256, 2017), clinical data of adult patients ( $>18$ years) who underwent elective valve surgery and/or coronary artery bypass grafting between July 1, 2011 and December 31, 2016 were screened. Exclusion criteria included aorta replacement, death within 24 hours after surgery and incomplete data. Primary outcome was postoperative death from all cause and requirement for the blood products during the hospital stay. The second outcome was ischemic events and bleeding related events after the surgery.

\section{RESULTS}

Of all eligible patients, 2,062 received TXA after CPB (the TXA group); 4,236 did not receive any TXA (the control group) (Figure 1). Incidences of postoperative death, ischemic events and bleeding related events were similar between groups. However, the requirements of any blood product in TXA was fewer than those in control group (Table 1). In multivariate logistic analysis, TXA after CPB reduced the risk of death, and exposure for any blood product, but not ischemic events and bleeding related events (Figure 2).

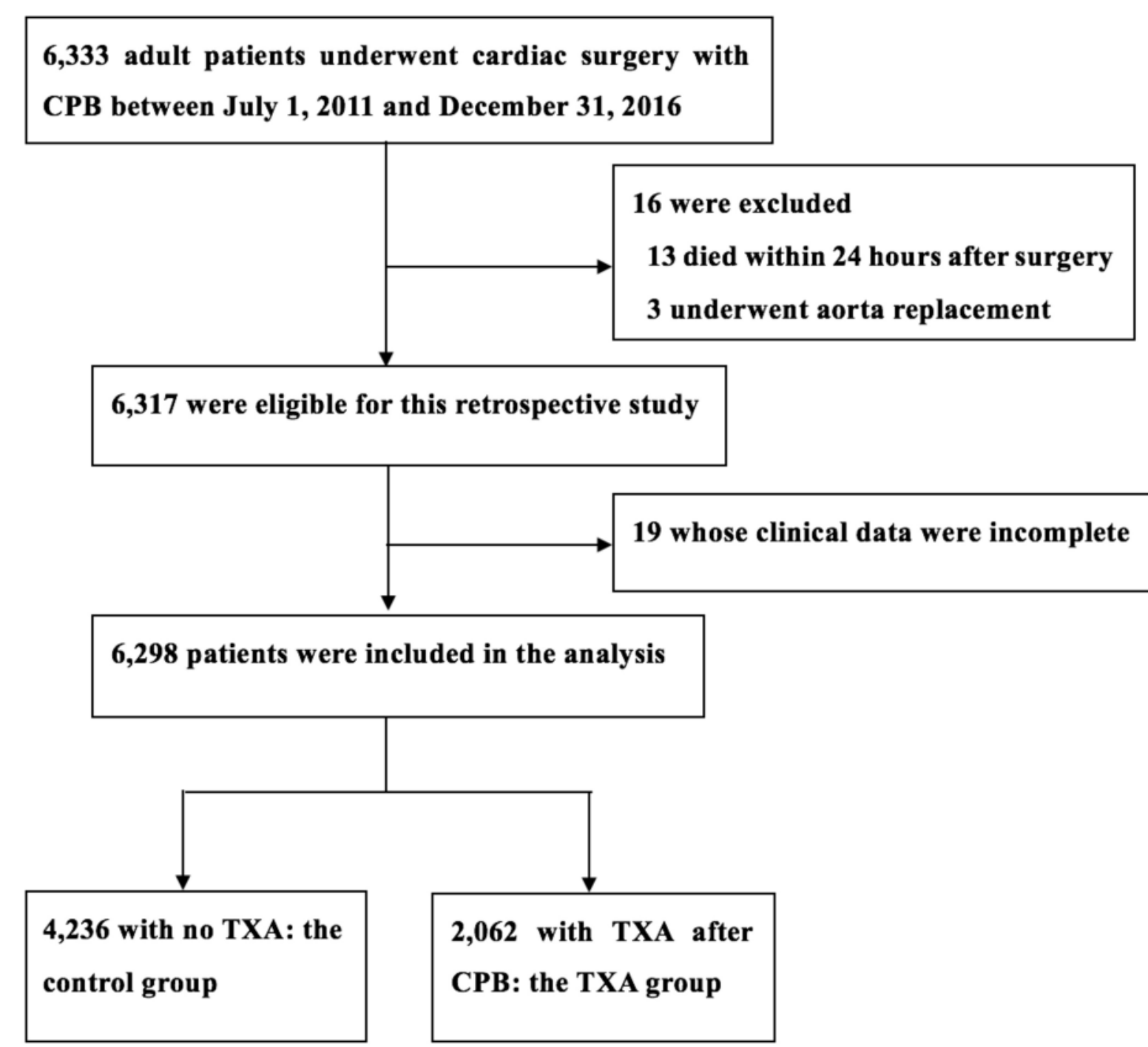

Figure 1. Patients screening

Table 1. Outcomes of the two groups

\begin{tabular}{|lccc|}
\hline Outcome & $\begin{array}{c}\text { No TXA } \\
(\mathrm{n}=4,236)\end{array}$ & $\begin{array}{c}\text { TXA } \\
(\mathrm{n}=2,062)\end{array}$ & P \\
\hline Any blood products & & & \\
Median (interquartile range) & $1.5(0,3.75)$ & $1.0(0,3.0)$ & 0.001 \\
Units per person & $2.74 \pm 5.21$ & $2.48 \pm 4.22$ & \\
$\mathrm{n}(\%)$ & $2428(57.2)$ & $1095(52.7)$ & 0.001 \\
Death, $\mathrm{n}(\%)$ & $61(1.4)$ & $18(0.9)$ & 0.058 \\
Ischemic events, $\mathrm{n}(\%)$ & $649(15.3)$ & $284(13.8)$ & 0.105 \\
Bleeding related events, $\mathrm{n}(\%)$ & $62(1.5)$ & $21(1.0)$ & 0.146 \\
\hline
\end{tabular}

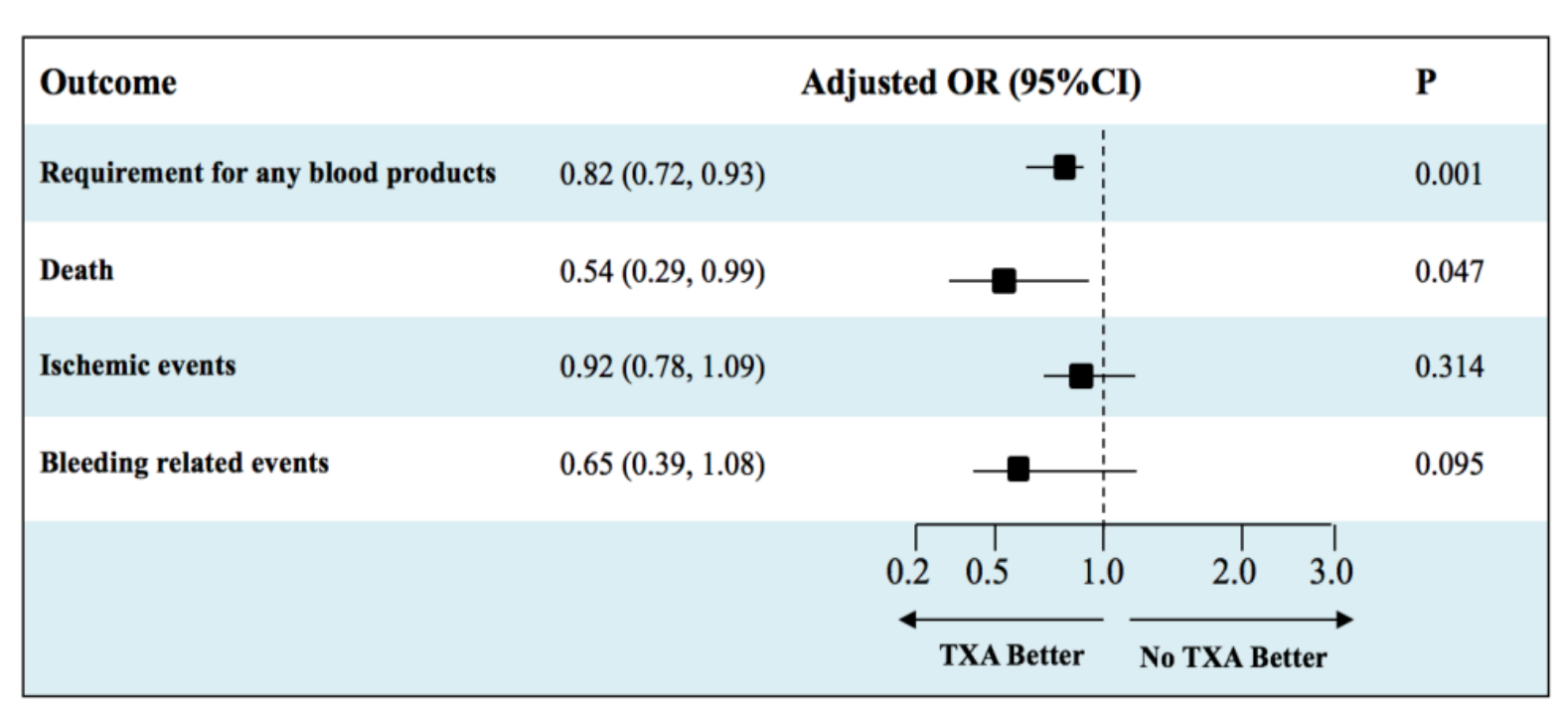

Figure 2. Relationship between TXA after CPB and outcomes

\section{CONCLUSIONS}

TXA after CPB reduced the risk of death and requirement for any blood product, but did not influence the ischemic events and bleeding related events. 